\title{
Die Entwicklung des OSZE-Instrumentariums für den Konfliktzyklus
}

\author{
Michael Raith"
}

\section{Zusammenfassung}

2021 wird der Ministerratsbeschluss Nr. 3/11 über Elemente des Konfliktzyklus zehn Jahre alt. Seit der Verabschiedung dieses zentralen Beschlusses arbeitet das Konfliktverhütungszentrum der OSZE daran, die Rolle der OSZE in relevanten Bereichen zu stärken, damit Konflikte nicht in Gewalt eskalieren, friedliche Konfliktlösungen erleichtert werden und die Schaffung von dauerhaftem Frieden und Sicherheit unterstützt wird. Die OSZE verfügt über ein umfangreiches Konfliktzyklusinstrumentarium, das im gesamten OSZE-Gebiet angewandt werden kann. Die Umsetzung der Instrumente kann kontinuierlich dadurch verbessert werden, dass Lehren aus vergangenem Handeln gezogen und künftige Herausforderungen vorhergesehen werden. Gleichzeitig sind jedoch dringend ausreichend Ressourcen für die OSZE erforderlich, sowie der politische Wille der Teilnehmerstaaten, die relevanten Instrumente auch proaktiv zu nutzen.

\section{Schlagworte}

OSZE, Konfliktverhütung, Frühwarnung, Krisenreaktion, Konfliktlösung, Mediationsunterstützung, Friedensförderung

Bitte zitieren als: Michael Raith, Die Entwicklung des OSZE-Instrumentariums für den Konfliktzyklus, OSCE Insights 3 (Baden-Baden: Nomos, 2021),

https://doi.org/10.5771/9783748911630-03

\section{Einführung}

Vor drei Jahrzehnten wurde mit der Charta von Paris für ein neues Europa das Konfliktverhütungszentrum $(\mathrm{KVZ})^{1}$ eingerichtet, welches später Teil des OSZE-Sekretariats wurde. Seither besteht die zentrale Rolle des KVZ darin, die OSZE-Teilnehmerstaaten dabei zu unterstützen, gewaltsame Konflikte zu verhüten oder gegebenenfalls friedlich bei-

* Dr. Michael Raith

OSZE-Konfliktverhütungszentrum, Wien Michael.Raith@osce.org zulegen und dauerhaften Frieden und nachhaltige Sicherheit zu schaffen. Eine weitere wichtige Säule des aktuellen OSZE-Instrumentariums kam 1992 mit dem Schlussdokument des Gipfeltreffens von Helsinki „Herausforderung des Wandels"2 hinzu, in dem die Teilnehmerstaaten vereinbarten, ihr gemeinsames Engagement bei der Verhütung und Lösung von Konflikten unter anderem durch die Einsetzung des Hohen Kommissars für Nationale Minderheiten (HKNM) zu verstärken. Der HKNM ist dazu beauftragt, die OSZE Teilnehmerstaaten zu informieren, wenn ethnische Spannungen zu eskalieren drohen, und übt somit eine 
der wichtigsten Frühwarnfunktionen der OSZE aus.

2011 wurde mit der Verabschiedung des Ministerratsbeschlusses Nr. 3/11 über Elemente des Konfliktzyklus ${ }^{3}$ ein weiterer Meilenstein erreicht. Seither baut die OSZE ihre Möglichkeiten im Bereich der Verhütung und Lösung von Konflikten, des Krisenmanagements, der Konfliktnachsorge und der Friedensförderung systematisch aus. Vor diesem Hintergrund befasst sich dieser Beitrag aus der Perspektive des KVZ mit der Entwicklung des Konfliktzyklus-Instrumentariums während der letzten zehn Jahre und macht Vorschläge, wie dieses in Zukunft weiter gestärkt werden kann.

\section{Konzeptionelle Grundlagen}

Das Konfliktzyklus-Instrumentarium basiert auf dem umfassenden Sicherheitsansatz der OSZE und geht daher davon aus, dass die Grundursachen von Konflikten und Instabilität in allen drei Dimensionen von Sicherheit liegen. Dazu gehören etwa militärische Bedrohungen zwischen Staaten, sozioökonomische Spannungen sowie Umweltzerstörung und Mängel in der Rechtsstaatlichkeit, um nur einige Punkte zu nennen. Die mehrdimensionale Kausalität von Konflikten bildet auch die Grundlage der Frühwarnmethodik der OSZE. ${ }^{4}$ Eine weitere wichtige konzeptionelle Grundlage dieses Instrumen- tariums ist ein „mehrstufiger Präventionsansatz": Primärprävention soll gewaltsame Konflikte verhüten, indem Instrumente zur Frühwarnung erfolgreich zum Einsatz kommen, frühzeitig gehandelt wird, und langfristige Maßnahmen, die an den strukturellen Konfliktursachen ansetzen, umgesetzt werden. Sekundärprävention greift, wenn ein Konflikt in Gewalt eskaliert. Dabei geht es um Krisenmanagement, um sowohl eine Intensivierung der Gewalt als auch deren geographische Ausweitung zu verhindern. Die Tertiärprävention, die gewöhnlich als Konfliktnachsorge und Friedensförderung bezeichnet wird, zielt darauf $a b$, erneute Spannungen und das Wiederaufflammen gewaltsamer Konflikte zu verhindern. Bemühungen um eine friedliche Konfliktlösung sind in allen drei Präventionsphasen erforderlich.

Die OSZE-Teilnehmerstaaten sind mit einem vielschichtigen Geflecht unterschiedlicher und sich überschneidender Sicherheitsherausforderungen und komplexer Konflikte konfrontiert, von denen viele weder ausschließlich innerstaatlich noch ausschließlich zwischenstaatlich sind. ${ }^{5}$ Dementsprechend beobachtet die OSZE ein breites Spektrum von Konfliktsituationen und eine zunehmende Anzahl transnationaler und regionaler Sicherheitsbedrohungen sowohl innerhalb des OSZE-Gebiets als auch in benachbarten Regionen. 
Abbildung 1: Der konzeptionelle Rahmen des KVZ zum Konfliktzyklus

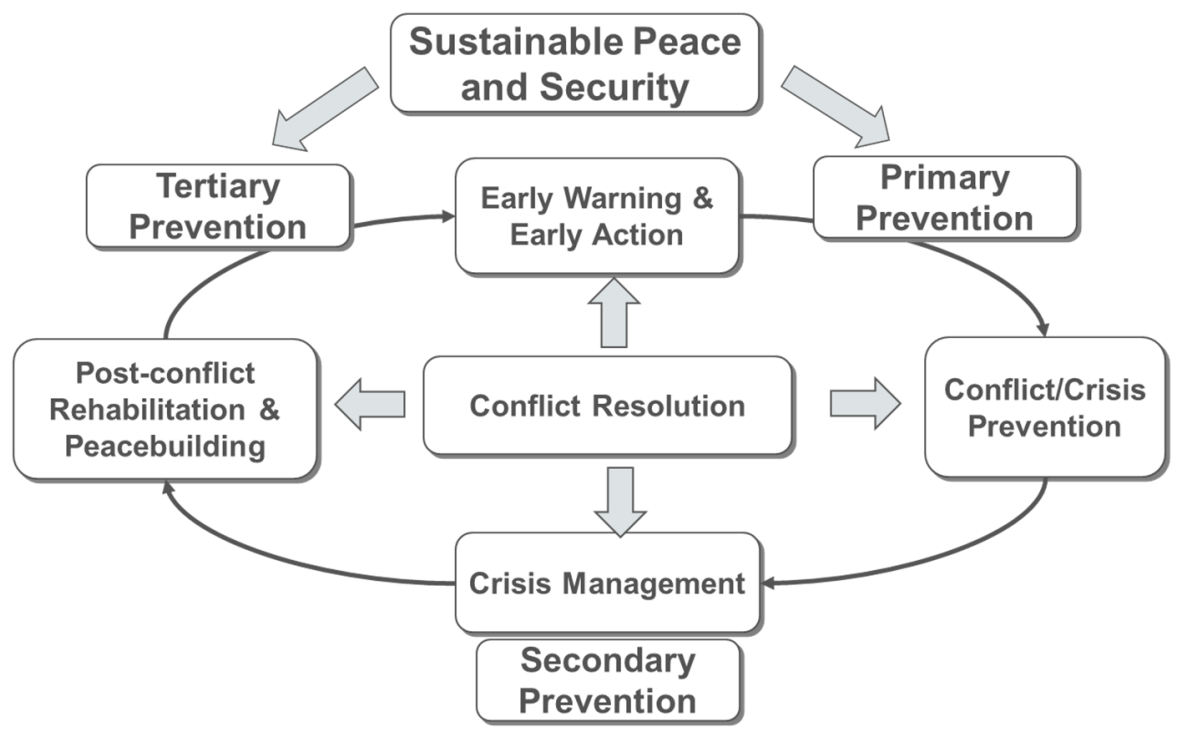

Der umfassende Sicherheitsansatz und der „mehrstufige Präventionsansatz" der OSZE ermöglichen hinreichende Flexibilität beim Umgang mit vielfältigen Konfliktsituationen. Diese Flexibilität ist in einem hochdynamischen Sicherheitsumfeld von entscheidender Bedeutung, nicht zuletzt deshalb, weil viele Konflikte sich nicht linear entwickeln, sondern zwischen latenten und akuten Krisenphasen abwechseln. Darüber hinaus können in ein und demselben Land oder in ein und derselben Region mehrere Konflikte mit unterschiedlicher Gewaltausprägung bestehen, sodass sich verschiedene OSZEAkteure gleichzeitig mit Konfliktverhütung, Krisenmanagement und Konfliktnachsorge befassen müssen. Daher ist die Koordination und Zusammenarbeit innerhalb und zwischen den unterschied- lichen OSZE-Durchführungsorganen besonders wichtig. Nur so kann sichergestellt werden, dass sich die jeweiligen Einsätze gegenseitig verstärken und ergänzen.

Der umfassende Sicherheitsansatz und der „mehrstufige Präventionsansatz“ der OSZE erfordern zudem einen ganzheitlichen Ansatz in der Friedensförderung, vergleichbar etwa mit jenem, der in der sogenannten „Agenda der Vereinten $\mathrm{Na}$ tionen zur Aufrechterhaltung des Friedens" abgedeckt ist. Dementsprechend sollten Instrumente der Friedensförderung, die eine gewaltfreie Konfliktlösung unterstützen und die Konfliktursachen an der Wurzel packen, während des gesamten Konfliktzyklus und nicht allein nach der Beendigung von Konflikten eingesetzt werden. 


\section{Die Signale wahrnehmen: Frühwarnung und Situational Awareness}

Effektive Frühwarnung ist eine Grundlage des Konfliktzyklus-Instrumentariums der OSZE, die den politischen Entscheidungsträgern analytische Beurteilungen von Risiken und Konfliktdynamiken liefert. Mithilfe von Frühwarnanalysen lassen sich Ansatzpunkte für kurzfristige operative Prävention identifizieren, um gewaltsame Eskalationen abzuwenden. Sie bietet darüber hinaus Möglichkeiten, langfristige strukturelle Prävention und Friedensförderung zu schaffen. Das betrifft etwa die demokratische Regierungsführung, Wirtschafts- und Umweltfragen, den Sicherheitssektor sowie den Schutz der Menschenrechte und Grundfreiheiten. All diese Bereiche werden von verschiedenen OSZE-Durchführungsorganen im Rahmen ihrer jeweiligen Mandate bearbeitet.

Im Einklang mit MR-Beschluss 3/11 fungiert das KVZ als OSZE-weite Anlaufstelle für Frühwarnung. Als solche erstellt es Frühwarnberichte für den OSZE-Vorsitz und den Generalsekretär und stärkt die Fähigkeiten der OSZE-Feldoperationen im Bereich der Frühwarnung und Konfliktanalyse. Das KVZ koordiniert darüber hinaus ein Netz von Anlaufstellen für Frühwarnung in allen Durchführungsorganen der OSZE, was ein entscheidender Vorteil für den Austausch von Informationen und Fachwissen ist. Das betrifft nicht nur den Bereich der Frühwarnung, sondern auch andere Instrumente des Konfliktzyklus wie die Förderung von Dialog, Mediation, Krisenmanagement, sowie Aussöhnung und
Friedensförderung. Das Netzwerk spielt eine zentrale Rolle bei der Stärkung einer Frühwarnkultur unter seinen Mitgliedern, indem es die Aufmerksamkeit der OSZE auf aktuelle Trends lenkt und sich abzeichnende Risikosituationen im Blick behält. Dazu gehören transnationale und regionale Sicherheitsbedrohungen sowie neuartige Herausforderungen wie die COVID-19-Krise, deren weitreichende Auswirkungen zu einem erhöhten Risiko politischer Instabilität und Spannungen in einigen Teilen des OSZE-Gebietes geführt haben.

Grundvoraussetzung für eine erfolgreiche Frühwarnung ist ein umfassendes Lagebewusstsein (Situational Awareness). Die OSZE verschafft sich dieses, indem sie relevante Entwicklungen in ihrem gesamten Gebiet und darüber hinaus verfolgt. Zwar profitieren die Frühwarnkapazitäten der Organisation immens von der Feldpräsenz der OSZE in Ostund Südosteuropa, im Südkaukasus und in Zentralasien. ${ }^{6}$ Wenn Frühwarnung jedoch effektiv sein soll, darf das Lagebewusstsein nicht nur auf die Gebiete begrenzt werden, in denen Feldoperationen stattfinden. Hier spielt der Situation Room des KVZ, der relevante Entwicklungen in allen 57 Teilnehmerstaaten und benachbarten Regionen der OSZE beobachtet, eine zentrale Rolle. Der Situation Room wertet rund um die Uhr offene Medienquellen aus und versorgt den Vorsitz, den Generalsekretär und die obere Führungsebene des Sekretariats mit aktuellen Nachrichten und regelmäßigen Updates. ${ }^{7}$ Im Krisenfall spielt der Situation Room eine wichtige Rolle in der Sicherheitskette zwischen Sekretariat und Feldoperatio- 
nen, was besonders außerhalb der offiziellen Arbeitszeiten von hoher Bedeutung ist.

Um den Informationsbedarf im $\mathrm{Zu}$ sammenhang mit aktuellen und neu entstehenden Sicherheitsherausforderungen zu verstehen, werden die Mitarbeiter"innen des Situation Room zu relevanten thematischen Fragen, wie zum Beispiel transnationale Bedrohungen, gebrieft. Der Situation Room verwendet, wenn möglich, auch neue digitale Technologien für die Beobachtung von öffentlich zugänglichen Quellen sowie für die tägliche Berichterstattung. Im heutigen komplexen Umfeld muss den sicherheitspolitischen Herausforderungen, die sich aus politischer Zersplitterung, Polarisierung, Nationalismus, Radikalisierung und Populismus ergeben, erhöhte Aufmerksamkeit geschenkt werden. Eine zunehmende Anzahl an Teilnehmerstaaten sind davon in unterschiedlichem Ausmaß betroffen. Darüber hinaus können im Zeitalter von Sozialmedien und Fake News latente Konflikte schneller als früher gewaltsam eskalieren. Unter der Oberfläche können sich Spannungen über einen langen Zeitraum aufbauen und sich dann plötzlich verursacht durch einen winzigen „virtuellen Funken“ entladen. Daher ist ein Situation Room mit ausreichenden Kapazitäten zur Beobachtung und Berichterstattung über solche Entwicklungen in Echtzeit heute wichtiger denn je.

Die Kapazitäten des Situation Room reichen derzeit nicht aus, um jeder Entwicklung im OSZE-Gebiet und seinen benachbarten Regionen die gleiche Aufmerksamkeit zu schenken. Der Austausch von Fachwissen, bewährten Verfahren und Informationen aus offenen Quellen mit den Krisen- und Lagezentren anderer internationaler Organisationen (wie den Vereinten Nationen und der Europäischen Union) ist daher von entscheidender Bedeutung und sollte so weit wie möglich gefördert werden. Darüber hinaus ist es erforderlich, dass systematisch Informationen von einer wachsenden Anzahl von Initiativen und Projekten eingeholt werden, die sich mit dem Mapping von Konflikten und Krisen befassen. ${ }^{8}$ Der MR-Beschluss 3/11 betont zudem, dass Frühwarnung auch in der Verantwortung der Teilnehmerstaaten liegt. Teilnehmerstaaten könnten daher relevante Informationen, die keiner Geheimhaltung unterliegen, mit dem Situation Room teilen, und somit die Frühwarnkapazitäten der OSZE stärken.

Mit MR-Beschluss 3/11 wird der Generalsekretär darüber hinaus beauftragt, dem Ständigen Rat der OSZE in Wien formelle Frühwarnungen zu übermitteln. Dieses Mandat ist eine bedeutende Weiterentwicklung, da die Übermittlung formeller Frühwarnsignale zuvor auf den HKNM beschränkt war. ${ }^{9}$ Trotz dieses Mandats hat der Generalsekretär seit 2011 nur zwei formelle Frühwarnungen an den Ständigen Rat ausgegeben. Die erste formelle Frühwarnung erfolgte im Dezember 2012 im Zusammenhang mit einer möglichen gewaltsamen Eskalation des Berg-Karabach-Konflikts, ${ }^{10}$ die zweite im November 2018 nach einem Zwischenfall zwischen russischen und ukrainischen Marineschiffen in der Straße von Kertsch. ${ }^{11}$

Formelle Frühwarnungen sollten ein letztes Mittel sein. Frühwarnung ist 
kein öffentliches Anprangern, sondern eine entscheidende Voraussetzung für wirksame Konfliktverhütung. Dementsprechend muss Frühwarnung in einer Art und Weise erfolgen, die keinen Schaden anrichtet und den Weg für präventive Bemühungen, einschließlich stiller Diplomatie, freimacht. Bevor eine formelle Frühwarnung ausgesprochen wird, werden der OSZE-Vorsitz und der Generalsekretär daher verschiedene andere Foren nutzen, um ihre Besorgnis über aufkommende Spannungen zum Ausdruck zu bringen und Diskussionen über präventive Maßnahmen zu ermöglichen. So wurde zum Beispiel der wöchentliche Bericht des Generalsekretärs an den Ständigen Rat bei zahlreichen Gelegenheiten genutzt, um eine Art informelle Frühwarnung zu übermitteln.

Einige Teilnehmerstaaten hätten im Rahmen der Frühwarnung gerne eine größere Sichtbarkeit des Generalsekretärs, auch was die Förderung des Austausches unter den Teilnehmerstaaten über mögliche Reaktionen betrifft. Im Einklang mit dem klaren Frühwarnmandat, das dem Generalsekretär im MR-Beschluss 3/11 erteilt wurde, könnte man darüber nachdenken, die Berichterstattung des Generalsekretärs an den Ständigen Rat um regelmäßige Frühwarnberichte zu erweitern, die dazu beitragen, dass Teilnehmerstaaten bereits bei sich abzeichnenden Trends - einschließlich transnationalen und regionalen Sicherheitsfragen - frühzeitig agieren können.

Eine zentrale Herausforderung für die OSZE-Frühwarnung und die entsprechende Rolle des Generalsekretärs ist das Problem der Politisierung. Zwar unter- stützten alle Teilnehmerstaaten im MRBeschluss 3/11 die Entwicklung eines systematischeren Ansatzes für Frühwarnung, jedoch befürworten nicht alle in gleicher Weise dessen praktische Umsetzung. Einige Teilnehmerstaaten würden vielleicht gerne von Beobachtungen für den Zweck der Frühwarnung ausgenommen werden, da sie diese als Stigma oder als Eingriff in die Souveränität betrachten.

Kein Teilnehmerstaat ist jedoch gegen Krisen immun. Jüngstes Beispiel dafür sind die Auswirkungen der COVID-19Pandemie. Daher müssen systematische Frühwarnbemühungen und Früherkennungsscans das gesamte OSZE-Gebiet und darüber hinaus abdecken, da sie für die Bewertung aufkommender Trends und künftiger Risiken von wesentlicher Bedeutung sind. ${ }^{12}$ Wenn das KVZ dem Generalsekretär und dem OSZE-Vorsitz zeitnahe Beurteilungen relevanter Entwicklungen vorlegt, legt es größten Wert darauf, eine Politisierung seiner Frühwarnanalysen zu vermeiden. Dementsprechend übermittelt das KVZ seine Frühwarnberichte und Ratschläge für frühzeitiges Handeln in vertraulicher Form. OSZE-Vorsitzende müssen die Frühwarnfunktion des KVZ aktiv nutzen und dem Generalsekretär und dem KVZ darüber hinaus die politische Unterstützung gewähren, die sie zur Erfüllung ihres Frühwarnmandats benötigen. ${ }^{13}$ Falls erforderlich, müssen sie dabei das „politische Feuer" auf sich lenken, um diesen entscheidenden Teil des Konfliktzyklus-Instrumentariums zu schützen. 
Frühzeitiges Handeln und

Krisenmanagement: Erkenntnisse für die Zukunft

Nach der Verabschiedung des MR-Beschlusses 3/11 wurden sofortige Schritte gesetzt, um die Krisenreaktionskapazitäten der OSZE zu stärken. Einer davon war die Einrichtung eines Virtual Pool of Equipment, um die rasche Verlegung wesentlicher Ausrüstungsgegenstände (wie zum Bespiel gepanzerter Fahrzeuge) zwischen OSZE-Feldoperationen zu ermöglichen. Ein weiteres Beispiel war das $R a$ pid Deployment Roster, das die rasche Entsendung von Einsatzpersonal aus unterschiedlichen Durchführungsorganen der OSZE ermöglicht, um neue Feldoperationen einzurichten oder bestehende vorübergehend zu verstärken. Darüber hinaus wurde das Operational Framework for Crisis Response entwickelt, das vom OSZE-Sekretariat genutzt werden soll, um im Einklang mit gewonnenen Erkenntnissen und bewährten Praktiken auf koordinierter Weise auf Krisen zu reagieren. Alle drei Instrumente hatten entscheidende Bedeutung bei der Planung und Entsendung der OSZE-Sonderbeobachtungsmission in die Ukraine (SMM) im März 2014. ${ }^{14}$

Die SMM ist die herausforderndste OSZE-Feldoperation seit der Kosovo-Verifizierungsmission im Jahr $1998,{ }^{15}$ die ebenfalls in einem komplexen Sicherheitsumfeld und im Kontext einer gewaltsamen Krise eingesetzt wurde. Die SMM begann als präventiver Einsatz mit der Aufgabe, die sich zunehmend verschärfende Sicherheitslage in der Ukraine zu beobachten, wurde aber schnell zu einer ausgewachsenen Krisenbewältigungsoperation. Die sich rapide verschlechternde Sicherheitslage im Osten der Ukraine führte zu völlig neuartigen operativen Risiken für die zivile Beobachtermission.

Die sich wandelnden Aufgaben und Aktivitäten der Mission, insbesondere die Beobachtung des Waffenstillstands in der Ostukraine, haben der OSZE eine steile Lernkurve abverlangt, die noch nicht völlig abgeflacht ist. ${ }^{16}$ Zusammen mit anderen Teilen des Sekretariats unterstützt das KVZ die SMM bei der kontinuierlichen Anpassung ihrer Operationen, ihres Personals und ihrer Ausstattung. Die Bereitstellung einer solchen Unterstützung erfordert jedoch große Ressourcen, und die Mitarbeiteranzahl innerhalb des KVZ ist gerade einmal das Minimum dessen, was notwendig ist, um die operative Unterstützung nicht nur der SMM, sondern aller 16 OSZE-Feldoperationen zu bewerkstelligen. ${ }^{17}$

Die SMM ist nicht nur eine Mission sui generis, sondern auch ein zentrales Beispiel für eine komplexe Friedensoperation. Als solche liefert sie viele wertvolle Erkenntnisse für die OSZE. Eine der wichtigsten Erkenntnisse für künftige Feldmissionen, die unter ähnlichen Umständen eingesetzt werden, ist die Tatsache, dass derartige Missionen ein anderes Personalmodell als die „traditionellen " OSZE-Feldoperationen erfordern, die sich meist auf programmatische und projektbezogene Aktivitäten konzentrieren. Komplexe Friedensoperationen erfordern robuste Management- und Führungsstrukturen sowie leitende Mitarbeiter"innen mit Erfahrung in Hochrisiko- 
gebieten. Sie benötigen zudem Personal mit Fachkenntnissen in Medizin, Technik, Infrastruktur und Minenräumung sowie hochqualifiziertes Sicherheitspersonal, das den grundlegenden Zusammenhang zwischen Einsatztätigkeiten und Sicherheit versteht.

Um von den Erfahrungen aus der anfänglichen Planung und Entsendung der SMM und der Ausweitung der Mission im Zuge der Minsker Abkommen zu profitieren, ${ }^{18}$ führte das KVZ 2015 eine umfassende Erkenntnisauswertung durch. ${ }^{19}$ Im Rahmen eines Auftrags des deutschen OSZE-Vorsitzes 2016 entwickelte das OSZE-Sekretariat unter Federführung des KVZ ein Kompendium von zwanzig internen Standard Operating Procedures (SOPs) für effektives Handeln in Situationen, die schnelle Reaktionen erfordern. Diese SOPs bieten bewährte Praktiken sowohl für neue als auch für bestehende Feldoperationen, unter anderem zur Missionsstruktur und Führung, zur operativen Berichterstattung und zum raschen Personaleinsatz. Auf der Grundlage der Erkenntnisse, die aus den Reaktionen der OSZE-Feldoperationen auf die COVID-19-Krise gewonnen wurden, könnten diese SOPs in Zukunft weiterentwickelt werden.

Die Erkenntnisse, die aus der SMM gewonnen wurden (und immer noch werden), beschränken sich nicht nur auf die Einrichtung neuer oder die Umstrukturierung bestehender Missionen unter veränderten Bedingungen vor Ort. Sie umfassen auch Erkenntnisse zu einem breiten Spektrum an neuen Technologien, die von der SMM im Rahmen ihrer Beobachtungstätigkeit angewandt werden. ${ }^{20}$
Durch die Nutzung von Satellitenbildern, akustischen Sensoren, Kamerasystemen und Drohnen im Rahmen einer zivilen Friedensoperation steht die OSZE in diesem Anwendungsgebiet nun in vorderster Reihe. Dies liefert viele wertvolle Erkenntnisse nicht nur für die Organisation, sondern auch für die internationale Gemeinschaft insgesamt. In diesem $\mathrm{Zu}-$ sammenhang stellt sich die Frage, wie die enormen Datenmengen, die mit technischen Beobachtungsmitteln gesammelt werden, verwaltet werden können. Das KVZ unterstützt daher die SMM auch bei der Erweiterung ihrer Kapazitäten zum Informationsmanagement, unter anderem bei der Implementierung eines missionsweiten Geoinformationssystems.

Die von der SMM etablierten bewährten Praktiken im Bereich technischer Beobachtung stellen eine wesentliche Verbesserung des Konfliktzyklus-Instrumentariums der OSZE dar. Um Erkenntnisse aus früheren und gegenwärtigen OSZEFeldoperationen einschließlich der SMM und ihrer technischen Innovationen zu gewinnen, entwickelt das KVZ derzeit einen Leitfaden zu Beobachtungstätigkeiten in allen Phasen des Konfliktzyklus. Er soll den umfassenden Erfahrungsschatz derjenigen OSZE-Missionen institutionalisieren, die zur Unterstützung ihres Mandats Beobachtungsaktivitäten durchführen. Darüber hinaus stellt das KVZ nun aufbauend auf den Erfahrungen der SMM eine/n Assistenz-Technologiereferent*in ein, der/die die OSZE-Abteilungen in technischen Fragen der Beobachtung und Überwachung unterstützen wird. ${ }^{21}$ 
Erkenntnisgewinn ist wichtig für künftige Krisenreaktionen. Krisenmanagement - wie im Osten der Ukraine sollte jedoch die Ausnahme bleiben, wohingegen die Verhütung gewaltsamer Krisen und die friedliche Lösung von Konflikten stets die Regel sein sollte. Konfliktverhütung erfordert erfolgreiches frühzeitiges Handeln, und das OSZE-Instrumentarium enthält hierzu eine Vielzahl bewährter Instrumente wie OSZEMechanismen und -Verfahren, ${ }^{22}$ vertrauensbildende Maßnahmen ${ }^{23}$ und Sonderbeauftragte oder Persönliche Beauftragte des/der Amtierenden Vorsitzenden der OSZE. Das letztere Instrument wurde zum Beispiel vom österreichischen OSZE-Vorsitz 2017 genutzt, um während der politischen Krise in der damaligen ehemaligen jugoslawischen Republik Mazedonien stille Diplomatie zu betreiben. Weitere wichtige Instrumente sind FactFinding Missionen, die mit Unterstützung des Gastlandes zur Informationsbeschaffung oder zur Dialogförderung, Vertrauensbildung und präventiven Diplomatie genutzt werden können. Ein präventiver Einsatz, der sowohl Elemente des Fact-Findings als auch der Dialogförderung umfasste, war das OSZE-Projekt zu einem Nationalen Dialog in der Ukraine im Frühjahr 2014. ${ }^{24}$ Ein weiteres Instrument war die Community Security Initiative der OSZE im Süden Kirgisistans, die von Oktober 2010 bis Dezember 2015 lief und einen wichtigen Beitrag zur Vertrauensbildung zwischen der Lokalbevölkerung und der Polizei lieferte. ${ }^{25}$

Ein zentrales Hindernis für eine erfolgreiche Konfliktverhütung und -lösung ist die Diskrepanz zwischen Frühwar- nung und frühzeitigem Handeln (Early Warning-Early Action Gap). In der Regel liegt dies nicht an einem Mangel an Frühwarnanalysen oder Instrumenten für frühzeitiges Handeln, sondern eher am fehlenden politischen Willen, die vorhandenen Krisenreaktionsinstrumente in vollem Umfang zu nutzen. Darüber hinaus kann die OSZE als Soft-Power-Organisation ihre Kapazitäten zur Krisenreaktion nur dann effizient einsetzen, wenn die lokalen Akteure vor Ort entgegenkommend handeln und kooperativ eingestellt sind.

Um die Lücke zwischen Frühwarnung und frühzeitigem Handeln zu schließen, entwickelte das KVZ 2018 die interne Early Warning-Early Action Matrix, die einen breiten Überblick über mögliche frühzeitige Maßnahmen bietet, die unter anderem vom OSZE-Vorsitz und OSZEDurchführungsorganen ergriffen werden können. Das KVZ betreibt auch eine Krisensimulation (SIMEX) für Teilnehmerstaaten die künftig den OSZE-Vorsitz übernehmen, um diese auf die praktische Anwendung der Krisenreaktionsinstrumente vorzubereiten. Anhand eines fiktiven Szenarios bietet die SIMEX zukünftigen OSZE-Vorsitzländern die Möglichkeit, die bestehenden OSZE-Instrumente und -Verfahren in einer sich entwickelnden Krise anzuwenden, und dabei Verantwortlichkeiten, Verfahren und Entscheidungsprozesse in einer Krisensituation abzuklären. 
Kernstück des Instrumentenkastens: Unterstützung friedlicher Konfliktlösung

Die Unterstützung einer friedlichen Lösung von Konflikten durch Mediation und Dialogförderung bildet das Herzstück des OSZE-Mandats. ${ }^{26}$ Mit ihrem umfassenden Sicherheitsansatz ist die OSZE in der einzigartigen Lage, den Dialog zu unterschiedlichen konfliktrelevanten Fragen auf kommunaler, regionaler und zwischenstaatlicher Ebene zu fördern. Angesichts der Komplexität heutiger Konflikte und Konfliktlösungsprozesse ist eine institutionalisierte, systematische und strukturierte Mediationsunterstützung mehr denn je erforderlich. ${ }^{27}$ Mediator*innen müssen mit der Komplexität der Konflikte, mit denen sie sich befassen, umgehen können, wenn sie die Parteien bei der Suche nach Gemeinsamkeiten und der Entwicklung gemeinsamer Lösungen unterstützen sollen. Deshalb benötigen Mediator"innen und ihre Teams engagierte Unterstützung bei der Konfliktanalyse, der Prozessgestaltung und den Micro-Skills, wie zum Beispiel Prozessanalyse- und Kommunikationstechniken.

Im MR-Beschluss 3/11 beauftragten die Teilnehmerstaaten den Generalsekretär mit der Ausarbeitung eines Vorschlags, „wie das Engagement der OSZE in Konfliktmediation möglichst nachhaltig, konsequent und wirksam gestaltet und die Rolle der OSZE-Mediatoren gestärkt werden kann." 28 Im Rahmen der Umsetzung dieses Auftrags wurde innerhalb des KVZ ein Mediationsunterstützungsteam (MST) eingerichtet, das aus drei Referent*innen für Mediationsunterstützung besteht und als OSZE-weite Anlaufstelle für Dialogförderung, Mediation und Mediationsunterstützung fungiert. ${ }^{29}$ Das MST ist heute ein unverzichtbares Instrument im Dienste der Stärkung der OSZE-Kapazitäten zur Förderung der friedlichen Lösung von Konflikten. Zur Unterstützung aller OSZEDurchführungsorgane und Teilnehmerstaaten befasst es sich vorwiegend mit folgenden Aufgaben: ${ }^{30}$

- Unterstützung der Entwicklung von Mediationsstrategien;

- Beratung zu Mediations- und Dialogprozessen;

- Unterstützung ausführlicher Konfliktanalysen;

- Bereitstellung von thematischen Briefings und Coaching für hochrangige OSZE-Mediator*innen, wie zum Beispiel Sonderbeauftragte des Amtierenden Vorsitzenden und Leiter*innen von OSZE-Feldoperationen;

- Förderung von Plattformen für den Austausch zwischen Mediator*innen;

- Debriefing mit OSZE-Mediator*innen, um Erfahrungen und Erkenntnisse über bewährte Praktiken zu gewinnen;

- Bereitstellung operativer Leitfäden zu mediationsrelevanten Themen;

- Training für OSZE-Mitarbeiter"innen im Bereich Mediation und Dialogförderung;

- Vermittlung von Experteneinsätzen zur Unterstützung von Mediationsprozessen.

Mit dem wachsenden Bedarf an Mediationsunterstützung verzeichnet das MST 
eine entsprechend steigende Nachfrage nach seinen Aktivitäten. Ein erweitertes MST würde es ermöglichen, hochrangigen Mediator*innen, die sich mit langwierigen Konflikten im OSZE-Gebiet befassen, spezifischere Mediationsunterstützung zukommen zu lassen. Auch die Aktivitäten von OSZE-Feldoperationen zur Dialogerleichterung und Mediation würden von einem erweiterten MST profitieren, da entsprechende Prozesse besser unterstützt werden könnten. Es könnte darüber hinaus Feldoperationen bei der Entwicklung ihrer eigenen Mediationsunterstützungskapazitäten stärker unter die Arme greifen. Für die SMM ist dies bereits geschehen. Durch die Aufstockung der Anzahl an Referenten für Mediation und Dialogförderung in Feldoperationen könnte ein OSZE-internes Netzwerk zur Mediationsunterstützung geschaffen werden.

Das MST sondiert immer neue Möglichkeiten zur Stärkung der OSZE-Konfliktlösungsinstrumente. Nach dem Ausbruch der COVID-19-Pandemie und dem Aufruf des UN-Generalsekretärs zu einem weltweiten Waffenstillstand stellte das MST Recherchen an und entwickelte einen strukturierten Ansatz zur Identifizierung von Chancen für Friedensprozesse. Dieser Ansatz bietet Ideen zur Prozessgestaltung, wie in Zeiten akuter Krisen eine positive Dynamik in laufenden Friedensprozessen erreicht werden kann. Ein weiterer Bereich der MST-Recherche unterstützt sogenannte Insider Mediator*innen, das heißt Mediator*innen, die in einem Konfliktkontext leben und ihn daher von innen kennen und möglicherweise einen besseren Zugang zu Konflikt- beteiligten haben als externe Dritte. ${ }^{31}$ Die Erkenntnis, dass lokale, einheimische und interne Methoden der Konfliktbehandlung Frieden und Stabilität langfristig stärken, rückt lokale Bemühungen zur Unterstützung von internen Mediator*innen in den Mittelpunkt der Friedensförderung.

Ein weiteres Thema, das im Zentrum der MST-Recherche steht, ist die Einbindung von Frauen in Friedensprozesse. Frauen erfahren Konflikte und Gewalt anders als Männer. Indem also die Perspektive der Frauen in die Konfliktlösungsbemühung einbezogen wird, können die unterschiedlichen Bedürfnisse der betroffenen Gesellschaften besser in Angriff genommen werden. Dementsprechend kann die Teilnahme von Frauen als Mediatorinnen und/oder Verhandlungsführerinnen in Friedensprozessen die Wahrscheinlichkeit, eine Einigung zu erzielen, verbessern und das Risiko eines Rückfalls in einen Konflikt verringern. $\mathrm{Zu}$ diesem Zweck haben das MST und die Abteilung für Genderfragen des Sekretariats 2019 ein Toolkit zum Thema „Einbindung von Frauen und effektive Friedensprozesse" entwickelt, das praktische Ratschläge und Empfehlungen enthält, wie mehr Gendersensibilität in Dialog- und Mediationsprozessen erreicht werden kann. ${ }^{32}$

\section{Nachhaltigkeit im Fokus: Strukturelle Prävention und Friedensförderung}

Die OSZE verfügt durch die langfristige programmatische Arbeit ihrer Durchführungsorgane über umfassende Erfah- 
rung im Umgang mit den strukturellen Ursachen von Konflikten und Instabilität. Dazu gehören die Bemühungen der drei autonomen OSZE-Institutionen, des Sekretariats und der OSZE-Feldoperationen. Mehr als 80 Prozent der OSZE-Mitarbeiter*innen sind im Feld tätig. Sie verwirklichen Projekte, mit denen die Umsetzung der OSZE-Prinzipien und -Verpflichtungen in allen drei Sicherheitsdimensionen unterstützt werden. Diese Aktivitäten zielen darauf $a b$, dauerhaften Frieden und nachhaltige Sicherheit zu schaffen, indem ein breites Spektrum an Themen umfassend gefördert wird. Dazu gehören etwa der Kampf gegen Radikalisierung und gewaltbereiten Extremismus, Menschenhandel, Hassverbrechen und Korruption sowie die Unterstützung der Bemühungen von Gastländern um gute Regierungsführung und Reformen im Zusammenhang mit Wahlen, Bildung und/oder dem Sicherheitssektor.

Dauerhaften Frieden und nachhaltige Sicherheit zu schaffen ist ein langfristiges Unterfangen, das Geduld, Ausdauer, umsichtige Planung und Weitsicht erfordert. Feldoperationen müssen sicherstellen, dass sich ihre Unterstützung an den sich entwickelnden Bedürfnissen und Prioritäten ihrer Gastländer orientiert. Dazu arbeitet die OSZE mit einer Vielzahl von Akteuren vor Ort zusammen, darunter Regierungsstellen, öffentliche Verwaltungen, die Zivilgesellschaft und Nichtregierungsorganisationen. Feldoperationen können entsprechend eine wichtige Brücke zwischen staatlichen und zivilgesellschaftlichen Akteuren schlagen und sie in gemeinsame Aktivitäten zur Unter- stützung der OSZE-Verpflichtungen und -Prinzipien einbinden.

Um Erkenntnisse aus der langjährigen Erfahrung von OSZE-Feldoperationen zu gewinnen, erstellte das KVZ 2018 ein Referenzhandbuch, das Einblicke in die Aktivitäten von OSZE-Feldoperationen zur strukturellen Prävention und Friedensförderung in Südosteuropa gibt. ${ }^{33}$ Das Referenzhandbuch hebt die Unterstützung der OSZE für Gastländer in sieben Themenbereichen hervor - Aufbau von Institutionen, gemeinschaftliche Sicherheit, Bildung, Wahlreform, Aussöhnung, Diversität sowie zwischenmenschliche Kontakte. Es zeigt auch auf, wie die Bemühungen der OSZE um Friedensförderung die Nachhaltigen Entwicklungsziele der Vereinten Nationen $(S D G s)^{34}$ ergänzen, insbesondere $S D G 16$ zur Förderung friedlicher und inklusiver Gesellschaften.

Eine zentrale Erkenntnis aus dem Referenzhandbuch ist, dass die Arbeit der OSZE dann am effektivsten ist, wenn sie kontextspezifisch und konfliktsensitiv umgesetzt wird und die historischen und gesellschaftlichen Entwicklungen vor Ort im Blick behält. Um die Fähigkeit der OSZE zur Schaffung dauerhaften Friedens und nachhaltiger Sicherheit zu verbessern, hat das KVZ gemeinsam mit dem Österreichischen Studienzentrum für Frieden und Konfliktlösung erstmalig einen OSZE-Kurs zur Friedensförderung für OSZE-Mitarbeiter"innen verschiedener Durchführungsorgane entwickelt. Hierbei geht es vor allem um die Stärkung der Fähigkeiten der OSZE, Grundursachen von Konflikten und In- 
stabilität in strukturierter und systematischer Weise anzugehen.

Ein wichtiger Bereich, in dem der Erfahrungsschatz der OSZE stärker zum Erkenntnisgewinn genutzt werden muss, ist das Gender-Mainstreaming langfristiger Konfliktverhütung- und Friedensförderungsmaßnahmen. Ein weiterer ist die Einbindung der Jugend in die Bewältigung aktueller und sich abzeichnender Sicherheitsherausforderungen, da junge Menschen positive gesellschaftliche Veränderungen beschleunigen können. Ein dritter Bereich von entscheidender Bedeutung für strukturelle Prävention und Friedensförderung ist die Unterstützung der OSZE für interessierte Teilnehmerstaaten im Bereich der Governance und Reform des Sicherheitssektors (SSG/R), zum Beispiel bezüglich der demokratischen Kontrolle der Streitkräfte. Das KVZ spielt bei diesen Bemühungen eine zentrale Rolle, unter anderem durch die Koordinierung eines Netzwerks von SSG/R-Anlaufstellen in den OSZE-Durchführungsorganen.

Obwohl unterschiedliche

OSZEDurchführungsorgane seit vielen Jahren Unterstützung im SSG/R-Bereich leisten, unternahm die OSZE erst 2014 erste Schritte zu einem systematischeren und strukturierteren Ansatz in diesem Bereich. Der damalige Schweizer OSZEVorsitz initiierte einen Prozess zur Erarbeitung von SSG/R-Leitlinien für OSZEMitarbeiter*innen, ${ }^{35}$ die 2016 veröffentlicht wurden. Gemeinsam mit den SSG/ $R$-Anlaufstellen in den Durchführungsorganen fördert das KVZ die Umsetzung dieser Leitlinien und hilft OSZE-Mitarbeiter*innen und -Missionsmitgliedern, die für die praktische Anwendung der Leitlinien erforderlichen Fähigkeiten zu entwickeln. Eine Überprüfung der Leitlinien ist derzeit im Gange, und eine zweite Ausgabe soll 2021 veröffentlicht werden.

SSG/R erfuhr ebenfalls besondere Aufmerksamkeit, als die Slowakei 2019 den Vorsitz der OSZE führte. Auf dem Ministerratstreffen in Bratislava hoben die Slowakei und 43 andere Teilnehmerstaaten die Bedeutung von SSG/R als einen entscheidenden Teil des umfassenden Sicherheitsansatzes der OSZE hervor. ${ }^{36} \mathrm{Da}-$ rüber hinaus berichtete der Generalsekretär den Teilnehmerstaaten im März 2019 über die seit 2014 unternommenen Anstrengungen zur Entwicklung eines kohärenteren und besser koordinierten SSG/R-Ansatzes, der auf den komparativen Vorteilen der OSZE aufbaut. ${ }^{37}$ Er betonte, dass das Konzept der SSG/R in zentralen OSZE-Prinzipien und -Verpflichtungen, wie etwa dem Verhaltenskodex zu politisch-militärischen Aspekten der Sicherheit (1994), verankert sei, und forderte, SSG/R stärker in das Konfliktzyklus-Instrumentarium zu integrieren und so den Mehrwert von SSG/R im Rahmen der Konfliktverhütung zu nutzen.

Ein rechenschaftspflichtiger und inklusiver Sicherheitssektor, der die Grundsätze der Rechtsstaatlichkeit achtet, kann wirksam zur Schaffung dauerhaften Friedens und nachhaltiger Sicherheit beitragen. SSG/R bleibt im Wesentlichen ein nationaler Prozess; die OSZE befindet sich jedoch in einer guten Position, um die Umsetzung nationaler Prioritäten zu unterstützen, insbesondere durch ihre Feldoperationen. Die OSZE kann 
auf eine lange Erfolgsbilanz bei der Unterstützung nationaler Institutionen des Sicherheitssektors und damit verbundener Akteure wie Parlamente und zivilgesellschaftliche Organisationen zurückblicken. Dementsprechend wird das KVZ weiterhin an einem kohärenten und systematischen SSG/R-Ansatz als zentrales Instrument der Friedensförderung innerhalb des Konfliktzyklus-Instrumentariums arbeiten. In diesem Zusammenhang können weitere Themen erörtert werden, wie zum Bespiel die Aufnahme SSG/R-relevanter Bestimmungen in Waffenstillstands- und Friedensabkommen und der Beitrag von SSG/R bei der Bearbeitung der Verknüpfung von (organisierter) Kriminalität und Konflikten.

\section{Austausch von Fachkenntnissen und Wissen: Nutzung von Partnerschaften}

Die Arbeit des KVZ zur weiteren Stärkung der OZSE-Kapazitäten in allen Phasen des Konfliktzyklus profitiert von starken Partnerschaften mit anderen internationalen und regionalen Organisationen. So tauscht sich das KVZ beispielsweise im Rahmen eines so genannten strukturierten Dialogs auf Arbeitsebene mit dem Auswärtigen Dienst der Europäischen Union (EAD) über Fachwissen und bewährte Praktiken in Bezug auf den Instrumentenkasten zum Konfliktzyklus aus. Das MST des KVZ steht darüber hinaus in regelmäßigem Kontakt mit den Einheiten für Mediationsunterstützung im EAD und im UN-Sekretariat. Durch die Partnerschaft mit der Einheit für Mediationsunterstützung der Verein- ten Nationen hat das MST Zugang zum UN-Standby-Team hochrangiger Mediationsberater, die zur Beratung in einem breiten Spektrum von Fragen der Mediation und präventiven Diplomatie rasch entsendet werden können. Regelmäßige und aktive Zusammenarbeit mit den Vereinten Nationen und dem EAD findet auch in Bezug auf SSG/R statt.

Die Zusammenarbeit der OSZE mit der UN-Hauptabteilung Unterstützung für Feldeinsätze führte 2017 zur Unterzeichnung einer Absichtserklärung, in der Kooperationsvereinbarungen zur Verbesserung der technischen Kapazitäten und des Fachwissens getroffen wurden. Aufgrund der Absichtserklärung kann die OSZE auf Vertragssysteme der Vereinten Nationen, zum Beispiel im Bereich des Beschaffungswesens, und auf technische Trainingsprogramme zugreifen, die einen kostengünstigen und raschen Zugang zu Ressourcen für frühzeitiges Handeln und Krisenreaktion ermöglichen.

Das KVZ unterhält zudem eine fruchtbare Zusammenarbeit mit dem UNFlüchtlingshilfswerk (UNHCR). Im Jahr 2014 veröffentlichte das KVZ zusammen mit dem UNHCR-Verbindungsbüro zur OSZE und den in Wien ansässigen UNOrganisationen eine Checkliste, die sich mit Vertreibung und dem Schutz vertriebener Bevölkerungsgruppen und betroffener Gemeinschaften in allen Phasen des Konfliktzyklus befasst. ${ }^{38}$ Seither bieten KVZ und UNHCR gemeinsam den Mitarbeiter*innen der OSZE und des UNHCR in Feldoperationen vor Ort Fortbildungskurse zur praktischen Anwendung der Checkliste an. 
Fazit

Das Konfliktzyklus-Instrumentarium der OSZE hat sich seit Anfang der 1990er Jahre erheblich weiterentwickelt. Die Verabschiedung und Umsetzung des MR-Beschlusses 3/11 waren in diesem Zusammenhang von entscheidender Bedeutung. Das Instrumentarium beinhaltet zuverlässige Instrumente, die im gesamten OSZE-Gebiet eingesetzt werden können. Die Umsetzung der Instrumente des Konfliktzyklus kann immer noch verbessert werden. Was jedoch dringend erforderlich ist, sind ausreichende OSZERessourcen und der politische Wille der Teilnehmerstaaten, die einschlägigen Instrumente und Mandate proaktiv zu nutzen. ${ }^{39}$

Angesichts der wachsenden Komplexität der heutigen Sicherheitsherausforderungen muss die OSZE bereit sein, einen sinnvollen Beitrag zur Konfliktverhütung und Friedensförderung zu leisten, wann immer und wo immer sie in ihrem weiten geographischen Raum dazu in der Lage ist. Dazu sind Flexibilität sowie ausreichende finanzielle und personelle Ressourcen erforderlich. Da die Kosten einer Krisenbewältigung die Kosten für präventive Maßnahmen bei weitem übersteigen, ${ }^{40}$ ergeben sich unzählige Vorteile aus Investitionen in Maßnahmen zur strukturellen Prävention und Friedensförderung. Dennoch war in den letzten Jahren ein kontinuierlicher Rückgang des Gesamthaushalts der OSZE zu verzeichnen, was die Fähigkeit der Organisation einschränkt, auf entstehende Spannungen zu reagieren und die strukturellen Ursachen von Konflikten zu be- arbeiten. Politischer Wille ist heute mehr denn je erforderlich, um das immense Potenzial der OSZE zu entfesseln und ihr Mandat und ihre Kapazitäten voll auszuschöpfen.

Der 10. Jahrestag des MR-Beschlusses 3/11 im Jahr 2021 während des schwedischen OSZE-Vorsitzes bietet eine ausgezeichnete Gelegenheit, eine Bestandsaufnahme des Konfliktzyklus-Instrumentariums vorzunehmen und dabei auch Möglichkeiten zu sondieren, die einschlägigen Instrumente weiter zu stärken und sie an neu entstehende Sicherheitsherausforderungen anzupassen. In diesem $\mathrm{Zu}$ sammenhang könnten die Teilnehmerstaaten die Verpflichtungen bekräftigen, die sie vor zehn Jahren im MR-Beschluss 3/11 eingegangen sind, zum Beispiel in einer Gedenkerklärung auf dem Ministerratstreffen 2021. Durch die Anerkennung der wichtigen Arbeit, die seit der Verabschiedung des MR-Beschlusses 3/11 geleistet wurde, würden die Teilnehmerstaaten den Anstrengungen der OSZE um eine Verbesserung des Konfliktzyklus-Instrumentariums neue Impulse geben und den Bemühungen in den Bereichen Konfliktverhütung, Konfliktlösung und nachhaltige Friedensförderung neuen Schwung verleihen.

\section{Notizen}

1 OSCE, Factsheet of the OSCE Conflict Prevention Centre, https://www.osce.org/ cpc/13717.

2 OSZE, Herausforderung des Wandels, 1992, https://www.osce.org/de/mc/3953 2. 
OSZE, Beschluss Nr. 3/11: Elemente des Konfliktzyklus im Zusammenhang mit der Verstärkung der Fähigkeiten der OSZE in den Bereichen Frühwarnung, frühzeitiges Handeln, Dialogerleichterung und Mediationsunterstützung sowie Konfliktnachsorge, MC.DEC/3/11, 7. Dezember 2011, https://www.osce.org/ de/ministerial-councils/86623.

OSCE, Early Warning: Internal OSCE Guidelines, 15 March 2012, interner Umlauf als SEC.GAL/52/12.

5 Vgl. in diesem Zusammenhang Sebastian von Einsiedel/Louise Bosetti/James Cockayne/Cale Salih/Wilfred Wan, Civil war trends and the changing nature of armed conflict, United Nations University Centre for Policy Research, March 2017, https://collections.unu.edu/eserv/UNU: 6156/Civil_war_trends_UPDATED.pdf.

OSCE, Survey of OSCE Field Operations, SEC.GAL/111/20/Corr.1*), 26 August 2020, https://www.osce.org/secreta riat $/ 74783$.

Zwischen Oktober 2018 und Oktober 2019 erstellte das Lagezentrum beispielsweise mehr als 500 Lagekurzberichte und mehr als 1.700 Kurzberichte zu speziellen Themen. Darüber hinaus versandte das Lagezentrum mehr als 11.000 E-MailBenachrichtigungen.

Vgl. z. B. Armed Conflict Location \& Event Data Project, „Dashboard“, https:// acleddata.com/dashboard/\#/dashboard.

Alice Ackermann, „Die Stärkung der Reaktion der OSZE auf Krisen und Konflikte: ein Überblick“, in: IFSH (Hrsg.), OSZE-Jahrbuch 2012, S. 228.

10 Claus Neukirch, „Frühwarnung und frühzeitiges Handeln - aktuelle Entwicklungen in der Konfliktverhütungstätigkeit der OSZE“, in: IFSH (Hrsg.), OSZEJahrbuch 2013, S. 136.

11 International Institute for Strategic Studies, „The Kerch Strait incident“, December 2018, https://www.iiss.org/publicatio ns/strategic-comments/2018/the-kerch-str ait-incident.

12 Vgl. in diesem Zusammenhang Lars Brozus, „The Difficulty of Anticipating Global Challenges: The Lessons of COVID-19“, in: Tom Bernes/Lars Brozus/Michal Hatuel-Radoshitzky et al., Challenges of Global Governance Amidst the COVID-19 Pandemic, Paper Series, Council on Foreign Relations, Mai 2020, 6-9, https://cdn.cfr.org/sites/ default/files/report_pdf/challenges-of-glo bal-governance-amid-the-covid-19-pande mic.pdf.

13 Nico Schernbeck, „Zwischen strategischer Neuausrichtung und operativen Anpassungen: aktuelle Herausforderungen und Möglichkeiten zur Stärkung von Frühwarnung und frühzeitigem Handeln in der Krisen- und Konfliktprävention der OSZE“, in: IFSH (Hrsg.), OSZE-Jahrbuch 2016, S. 158.

14 Claus Neukirch, „Die Sonderbeobachtermission in der Ukraine: operative Herausforderungen und neue Horizonte", in: IFSH (Hrsg.), OSZE-Jahrbuch 2014, $207 \mathrm{ff}$.

15 OSCE, „OSCE Kosovo Verification Mission/OSCE Task Force for Kosovo (closed)“, https://www.osce.org/kvm-closed.

16 Marcel Peško, „Die Reaktion der OSZE auf die Krise in der Ukraine: neue Lösungen für neue Herausforderungen “, in: IFSH (Hrsg.), OSZE-Jahrbuch 2016, $25-$ 36.

17 Gegenwärtig sind im KVZ Operations Service zwei Stellen für die operative Planung abgestellt.

18 Heidi Tagliavini, „Mediation während der Krise in der Ostukraine bis zum 23. Juni 2015“, in: IFSH (Hrsg.), OSZEJahrbuch 2015, 239-251.

19 Zusätzlich zu diesen spezifischen Aktivitäten führt das KVZ auch regelmäßige Nachbesprechungen am Ende des Einsatzes aller scheidenden Leiter*innen und stellvertretenden Leiter"innen von 
OSZE-Feldoperationen durch. Die Ergebnisse dieser Nachbesprechungen sind für die Pflege des institutionellen Gedächtnisses der OSZE von zentraler Bedeutung.

20 Cono Giardullo/Walter Dorn/Danielle Stodilka, „Technological Innovation in the OSCE: The Special Monitoring Mission in Ukraine", in: IFSH (eds.), OSCE Yearbook 2019, 119-137.

21 OSCE, „Associate Technology Officer“, https://jobs.osce.org/vacancies/associate-t echnology-officer-vnsecs01548.

22 OSCE, OSCE Mechanisms and Procedures: Summary/Compendium, 2011, https://www.osce.org/cpc/34427.

23 OSCE, OSCE Guide on Non-military Confidence-Building Measures, 2012, https://www.osce.org/secretariat/91082

24 OSCE, „OSCE National Dialogue Project completes field work in Ukraine“, 17 April 2014, https://www.osce.org/ukrai ne/117808.

25 OSCE, „CSI: Kyrgyzstan - The Community Security Initiative“, 10 March 2015, https://www.osce.org/bishkek/144296.

26 OSCE, Mediation and Dialogue Facilitation in the OSCE, https://www.osce.org/s ecretariat/126646.

27 Christina Stenner, The institutionalization of mediation support: Are mediation support entities there yet?, Berghof Foundation, 2017, https://www.berghof-found ation.org/fileadmin/redaktion/Publicatio ns/Other_Resources/Stenner_TheInstitut ionalizationOfMediationSupport.pdf.

28 Beschluss Nr.3/11 über Elemente des Konfliktzyklus, oben zitiert (Anm.3), S. 5 .

29 David Lanz/Jamie Pring/Corinne von Burg/Mathias Zeller, Understanding mediation support structures, swisspeace, October 2017, https://www.swisspeace. ch/fileadmin/user_upload/pdf/Mediation /swisspeace_MSS_study_18_Oct_Final.p df.
30 OSCE, „Mediation and mediation support", https://www.osce.org/secretariat/ 107488.

31 OSCE/Berghof Foundation, OSCE Support to Insider Mediation: Strengthening Mediation Capacities, Networking and Complementarity, December 2016, https://www.osce.org/support-to-insider -mediation.

32 OSCE, Inclusion of Women and Effective Peace Processes: A Toolkit, 2019, https://www.osce.org/secretariat/44073 5.

33 OSCE, Building Sustainable Peace and Democracy: OSCE Experiences in SouthEastern Europe, 2018, https://www.osce.o $\mathrm{rg} /$ secretariat/383751.

34 Thomas Greminger, „Sustaining Peace, Sustaining Development - The Role of the OSCE“, in: IFSH (ed.), OSCE Yearbook 2019, 27-39.

35 OSCE, Security Sector Governance and Reform: Guidelines for OSCE Staff, 2016, https://www.osce.org/secretariat/ 231176.

36 Permanent Mission of the Slovak Republic to the Organization for Security and Co-operation in Europe, Statement on the OSCE's Role in Supporting Security Sector Governance and Reform as Part of the OSCE's Comprehensive Approach to Security, MC.DEL/63/19, 16 December 2019, https://www.osce.org/ministerial -councils/442288.

37 OSCE, Report by the Secretary General on the OSCE Approach to Security Sector Governance and Reform (SSG/R), SEC.GAL/42/19, 14 March, https://www .osce.org/secretary-general/414725.

38 OSCE/UNHCR, Protection Checklist: Addressing Displacement and Protection of Displaced Populations and Affected Communities along the Conflict Cycle: A Collaborative Approach, 2014, https:// www.osce.org/secretariat/111464.

39 OSCE, A Stronger OSCE for a Secure Europe: Further Strengthening OSCE 
Capabilities and Capacities across the Conflict Cycle, Report by the German OSCE Chairmanship 2016 to the Ministerial Council, MC.GAL/5/16, 8 December 2016, https://www.osce.org/cio 1287431 .
40 Vereinte Nationen/Weltbankgruppe, Pathways for peace: inclusive approaches to preventing violent conflict, 2018, https://openknowledge.worldbank.org/ handle/10986/28337. 